\title{
A Case Report of Fatal Outcome of Cerebral Venous Sinus Thrombosis With Cerebral Hemorrhage During Early Pregnancy Secondary to Antithrombin III Deficiency
}

\author{
Nurezwana Elias $^{{ }^{*}}$, Kavitha MurajRajamany ${ }^{1}$, Noor Azmi Mat Adenan ${ }^{1}$
}

\begin{abstract}
Objectives: Cerebral venous sinus thrombosis (CVST) with cerebral hemorrhage is relatively rare but may result in a catastrophic outcome. Antithrombin III deficiency is an inherited disease that contributes to an increase in the occurrence of thromboembolic events during pregnancy.

Case Presentation: A nulliparous, 34-year-old Chinese woman at 9 weeks of pregnancy with underlying antithrombin III deficiency was under treatment with low dose molecular weight heparin. She presented with a frontal headache and projectile vomiting. A plain computed tomography (CT) scan and magnetic resonance angiography and venography (MRAV) of the brain showed features suggestive of CVST involving superior sagittal sinus with venous infarct and right frontal and left parietal intraparenchymal hemorrhage. During admission, she developed episodes of fainting and loss of consciousness. A repeated brain CT scan showed worsening intraparenchymal hemorrhages with midline shift, subfalcine herniation and cerebral edema. An emergency bilateral craniectomy was performed to relieve the intracranial pressure. Despite the surgery and supportive measures, the patient succumbed to death.

Conclusion: Due to the highly hypercoagulable state of pregnancy with concomitant antithrombin III deficiency, preconception counselling including optimization of anticoagulant dosage is crucial. In addition, the administration of antithrombin III concentrate should be considered during pregnancy for women with antithrombin deficiency.

Keywords: Sinus thrombosis, Intracranial, Antithrombin III deficiency, Pregnancy, Anticoagulants
\end{abstract}

\section{Introduction}

Antithrombin III deficiency is a rare hereditary thrombophilia. It is associated with 2-4 fold higher risks for venous thrombosis and starts as early as 21-40 years old. By the age of 50 years, approximately $50 \%$ of affected individuals would have experienced the first episode of thrombosis, with women having an earlier average peak incident compared to men $(1,2)$.

Thrombosis in patients with antithrombin III deficiency predominantly occurs in venous circulation. Commonly, the thrombosis occurs at deep veins of limbs with subsequent embolus to pulmonary circulations, but it can also occur in unusual sites, such as cerebral, retinal, mesenteric, portal, hepatic, and splenic veins (3).

Women with hereditary thrombophilia during pregnancy have higher risks of thrombosis if no thromboprophylaxis is given. The prevalence of a thrombosis seems to be higher during the first trimester of pregnancy as compared to advanced stages of pregnancy or puerperium (4).

Here, we report a case of massive cerebral venous sinus thrombosis (CVST) with intracerebral hemorrhage in a young woman in her first trimester pregnancy, with underlying antithrombin III deficiency that leads to a fatal maternal outcome.

\section{Case Report}

A 34-year-old Chinese woman, nulliparous at 9 weeks of pregnancy with underlying hereditary antithrombin III deficiency presented with severe bilateral frontal headache worsening over 5 days. This was accompanied by projectile vomiting, lethargy, generalized body weakness, and feeling drowsy over the same duration.

She had a history of pulmonary embolism at the age of 15 and was found to have antithrombin III deficiency. The family screening was done and all her siblings were found to have antithrombin III deficiency. Her brother died due to pulmonary embolism at the age of 30 . She was then put on lifelong vitamin $\mathrm{K}$ antagonist (warfarin).

In December 2015, her anticoagulant was switched to low molecular weight heparin; enoxaparin $40 \mathrm{mg}$ daily after she was found to be pregnant to avoid risks 
of warfarin teratogenicity. Unfortunately, the pregnancy ended in miscarriage and she was later put back on warfarin. In October 2016, her anticoagulant was changed to tinzaparin, a low molecular weight heparin with the dosage of 9000 units daily (175 units/kg), as patient wanted to conceive again. She conceived spontaneously within 6 months and was in good health until she developed the symptoms 5 days prior to her admission.

Whilst waiting to be admitted, she developed multiple episodes of generalized tonic-clonic seizure with altered mental status. During the reexamination, she was drowsy with a Glasgow Coma Scale of E3V5M4 and her pupils were reactive bilaterally. She was normotensive, afebrile, and had a pulse rate of 100 beats/min. A plain computed tomography (CT) scan of the brain showed right frontal and left parietal intraparenchymal hemorrhage with superior sagittal sinus thrombosis. Magnetic resonance angiography and venography (MRAV) were performed which showed thrombosis of the superior sagittal sinus with venous infarct and intraparenchymal hemorrhage with midline shift as shown in Figure 1.

Her antithrombin III level was 36\% and anticoagulant was withheld in view of cerebral hemorrhages. She underwent emergency bilateral frontal decompressed craniectomy surgery to relieve the intracranial pressure. During the operation, her brain did not appear pulsatile upon entering the dura. Blood clots were not removed to prevent further hemorrhage.

Her condition worsened postoperatively with her intracranial pressure remained high. A repeated CT scan of the brain showed a progressive worsening of intracranial hemorrhage. She developed diabetes insipidus and multiorgans dysfunction and later succumbed to death despite all supportive measures.

\section{Discussion}

Pregnancy is a prothrombotic state, as during this period there is a marked existence of all 3 constituents of Virchow's triad: hypercoagulable state, endothelial injury, and stasis of venous circulation. This condition may be worsened by the presence of a hereditary coagulable condition such as antithrombin III deficiency, a rare hereditary thrombophilia. In thrombophilia, the balance between "pro-coagulant" and "anticoagulant" activity is constantly disrupted. The lifetime risk of experiencing thrombotic events for those with such a condition is 16 times higher than those without thrombophilia (5).

Preconception counselling is important for women with thrombophilia who wish to become pregnant. This intervention can help to improve health outcomes for women, newborns and children. Sadly, the awareness regarding the preconception care among health provider as well as among patients remains oblivious. Around $38 \%$ of births worldwide are unplanned (6), while in the United States fewer than half of couples have even heard of preconception counselling (7).

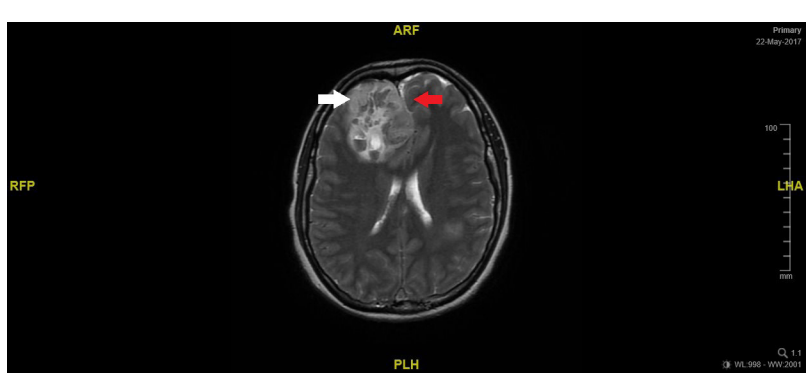

Figure 1. Transverse view of MRAV of the brain. White arrow indicates right temporal lobe hemorrhage. Red arrows indicates midline shift.

Explanation regarding the risk of thromboembolic event and transmission to their offspring is vital to ensure compliance to follow up and treatment. The management of anticoagulant drugs and their dosage should ideally be a combined care from a hematologist and maternal medicine consultants. Patients should be explained on the need for treatment, the risks of treatment versus no treatment to the mother and fetus, types of drugs available and degree of monitoring needed. There are many available anticoagulant agents considered safe in pregnancy, but some agents may have adverse effects on the mother and/or fetus (8).

In pregnant women with antithrombin III deficiency, administration of antithrombin III concentrate in addition to anticoagulation is recommended (9), particularly in patients who develop venous thromboembolism despite being on therapeutic dose anticoagulation (10). This is because patients with antithrombin III deficiency may be heparin resistant, needing higher heparin doses to achieve therapeutic activated partial thromboplastin time (aPTT) and protective anticoagulation (11). Unfortunately, antithrombin III concentrate is not easily or widely available, especially in Malaysia. Administration of antithrombin III concentrate during an acute thrombosis event might be beneficial for this case.

A thrombosis event such as CVST may result in a devastating outcome if it is extensive and associated with cerebral hemorrhage, as highlighted in this case. The diagnosis can be easily confused with other common causes of headache or seizure in pregnancy which have similar clinical features (12). Thus, the presence of any neurological symptoms, however mild, is sufficient to prompt further investigations. Clinicians should be alerted to the onset of neurological symptoms and differentiate them from early pregnancy symptoms. Patients should be advised to seek early medical attention and not to confuse thrombosis events with early pregnancy symptoms, as demonstrated in this case. The patient had experienced headache and vomiting for 5 days and assumed that the symptoms were due to morning sickness. Therefore, failure to recognize early neurological symptoms led to a fatal outcome. The management of CVST is mainly the treatment of underlying causes and prevention of 
complications such as cerebral hemorrhage that can cause an increase in intracranial pressure (13).

In conclusion, antithrombin III deficiency is not common and may be confused with early pregnancy symptoms. Optimum combined care involving multidisciplinary teams with an emphasis on early recognition of neurological symptoms should be highlighted during preconception counselling for women at high risk of developing cerebral thrombosis. Antithrombin III concentrate should be made available for the benefit of the patients.

\section{Conflict of Interests}

Authors declare that they have no conflict of interests.

\section{Ethical Issues}

Written informed consent was obtained from the patient for publishing this case.

\section{Financial Support}

None.

\section{References}

1. Bucciarelli P, Rosendaal FR, Tripodi A, et al. Risk of venous thromboembolism and clinical manifestations in carriers of antithrombin, protein $\mathrm{C}$, protein $\mathrm{S}$ deficiency, or activated protein $\mathrm{C}$ resistance: a multicenter collaborative family study. Arterioscler Thromb Vasc Biol. 1999;19(4):10261033. doi:10.1161/01.ATV.19.4.1026

2. James AH, Konkle BA, Bauer KA. Prevention and treatment of venous thromboembolism in pregnancy in patients with hereditary antithrombin deficiency. Int J Womens Health. 2013;5:233-241. doi:10.2147/ijwh.s43190

3. Patnaik MM, Moll S. Inherited antithrombin deficiency: a review. Haemophilia. 2008;14(6):1229-1239. doi:10.1111/ j.1365-2516.2008.01830.x

4. van Boven HH, Vandenbroucke JP, Briët E, Rosendaal FR. Gene-gene and gene-environment interactions determine risk of thrombosis in families with inherited antithrombin deficiency. Blood. 1999;94(8):2590-2594.

5. Vossen CY, Conard J, Fontcuberta J, et al. Familial thrombophilia and lifetime risk of venous thrombosis. J Thromb Haemost. 2004;2(9):1526-1532. doi:10.1111/ j.1538-7836.2004.00852.x

6. Sedgh G, Singh S, Hussain R. Intended and unintended pregnancies worldwide in 2012 and recent trends. Stud Fam Plann. 2014;45(3):301-314. doi:10.1111/j.17284465.2014.00393.x

7. Mitchell EW, Levis DM, Prue CE. Preconception health: awareness, planning, and communication among a sample of US men and women. Matern Child Health J. 2012;16(1):31-39. doi:10.1007/s10995-010-0663-y

8. Alshawabkeh L, Economy KE, Valente AM. Anticoagulation During Pregnancy: Evolving Strategies With a Focus on Mechanical Valves. J Am Coll Cardiol. 2016;68(16):18041813. doi:10.1016/j.jacc.2016.06.076

9. Bramham K, Retter A, Robinson SE, Mitchell M, Moore GW, Hunt BJ. How I treat heterozygous hereditary antithrombin deficiency in pregnancy. Thromb Haemost. 2013;110(3):550-559. doi:10.1160/th13-01-0077

10. Refaei M, Xing L, Lim W, Crowther M, Boonyawat K. Management of Venous Thromboembolism in Patients with Hereditary Antithrombin Deficiency and Pregnancy: Case Report and Review of the Literature. Case Rep Hematol. 2017;2017:9261351. doi:10.1155/2017/9261351

11. Chryssos A, Stroup SJ, Pifer MM, Tawil M, Conrad CG. Management of Heparin-Resistant Patients with Benefits? Maximizing Biocompatibility in Cardiopulmonary Bypass: Combining ATryn(R) Recombinant Antithrombin III and Carmeda(R) Heparin-Bonded Perfusion Circuits: A Case Series. J Extra Corpor Technol. 2015;47(1):44-47.

12. de Bruijn SF, Stam J, Kappelle LJ. Thunderclap headache as first symptom of cerebral venous sinus thrombosis. CVST Study Group. Lancet. 1996;348(9042):1623-1625.

13. Benveniste RJ, Patel AB, Post KD. Management of Cerebral Venous Sinus Thrombosis. Neurosurg Q. 2004;14(1):27-35. doi:10.1097/00013414-200403000-00005

(c) 2018 The Author (s); This is an open-access article distributed under the terms of the Creative Commons Attribution License (http://creativecommons.org/licenses/by/4.0), which permits unrestricted use, distribution, and reproduction in any medium, provided the original work is properly cited. 